\title{
Benefits of visco-acoustic full waveform inversion in Foz do Amazonas basin
}

Debora Mondini, Edmarley Ramos, Karine Pereira, Erick Tomaz, Daniela Donno, Diego Carotti, CGG

Copyright 2019, SBGf - Sociedade Brasileira de Geofísica

This paper was prepared for presentation during the $16^{\text {th }}$ International Congress of the Brazilian Geophysical Society held in Rio de Janeiro, Brazil, 19-22 August 2019.

Contents of this paper were reviewed by the Technical Committee of the $16^{\text {th }}$ International Congress of the Brazilian Geophysical Society and do not necessarily represent any position of the SBGf, its officers or members. Electronic reproduction or storage of any part of this paper for commercial purposes without the written consent of the Brazilian Geophysical Society is prohibited.

\begin{abstract}
Absorption is a well-known source of amplitude loss and phase distortion in seismic data. For proper imaging, both the velocity and the quality factor $(Q)$ models need to be accurately estimated. Ray-tracing-based Q-tomographic inversions can perform well, but typically provide lower resolution than a full-waveform inversion approach. In addition, the tomography method has inherent drawbacks in shallow-water datasets, due to acquisition limitations and its reliance on reflection data. Advances in fullwaveform inversion technology have recently offered a new possibility to compensate for phase distortion and amplitude loss by a joint update of velocity and $Q$. Several industrial applications on towed-streamer data have recently been published. In this paper, we present the application of this technology on a shallow-water dataset offshore Brazil affected by strong absorption in the overburden. We show that our Q-FWI can invert for highresolution velocity and attenuation models, providing superior imaging when coupled with an attenuation compensating pre-stack depth migration (Q-PSDM).
\end{abstract}

\section{Introduction}

Absorption has two different effects on seismic imaging: it creates phase distortion that is responsible for incorrect positioning of the seismic events and subsequent loss of resolution due to reduced focusing, and amplitude attenuation caused by high frequency energy loss. In conventional seismic imaging, absorption is usually compensated by applying a constant quality factor (Q) correction for both phase distortion and amplitude loss. However, in complex geological regions, for example in presence of shallow gas pockets, the use of a constant correction yields underestimation of the absorption effects, resulting in a seismic image with poor resolution and unbalanced amplitudes.

Therefore, it is necessary to estimate a $3 D$ volumetric $Q$ to effectively compensate for the absorption and to obtain a more interpretable image. Methods such as ray-based Qtomography have proven to be able to estimate the lateral variations of the background $Q$ and to localize some $Q$ anomalies (Hung et al., 2008; Gamar et al., 2015). The resultant $Q$ model is conformal to the geology, however it is sometimes characterized by a low spatial resolution. One important limitation for $Q$ tomography is that it relies on reflections. This constitutes a challenge for shallow water datasets due to the suboptimal illumination in the shallow region.

In case of shallow complex geometries, visco-acoustic fullwaveform inversion (Q-FWI) could be a reasonable choice for estimating high-resolution velocity and absorption models, since it relies on diving waves. Despite the wellknown challenge of inverting more than one parameter with FWI, the joint inversion of velocity and Q (Wang et al., 2018), using a large bandwidth of data, has shown to help in mitigating the cross-talk between these two parameters (Plessix et al., 2016). Successful examples of Q-FWI applied on field data have been reported by Malinowski et al. (2011), Stopin et al. (2016) and Xiao et al. (2018).

We applied Q-FWI using the formulation given by Wang et al. (2018) in a shallow area of the Foz do Amazonas basin, offshore Brazil. Compared with the Q-tomography results, this method allows identification of shallow absorption bodies with high resolution that are highly consistent with the seismic structures, providing an uplift in the imaging process.

\section{Method}

Among the various methods to estimate volumetric $Q$ models, we choose the frequency-peak shift (FPS) method proposed by Gamar et al. (2015). Through the estimation of the FPS, a four-dimensional volumetric effective $Q$ volume (time, inline, crossline, and offset) was obtained and then used within $Q$ tomography to obtain a $3 D$ interval $Q$ model. Together with the $3 D$ volumetric $Q$ model, a TTI model was built through several iterations of multi-layer tomography (Guillaume et al., 2012).

We then applied Q-FWI, with the aim of improving the lowresolution tomography-based $Q$ model. Wang et al. (2018) have presented the extension of FWI technology to invert for velocity and $Q$. The joint inversion of velocity and $Q$, over a large frequency bandwidth of data, allows better handling of the cross-talk between these two parameters. For strong anomalies, the differences between the effects of velocity and $Q$ become apparent when considering a relatively broad range of frequencies, thus enabling Q-FWI to create meaningful updates (Plessix et al., 2016).

The input data for Q-FWI was pre-processed by removing spikes and swell noise. For the source wavelet, the nearfield hydrophone measurements were inverted to derive a far-field signature (Poole et al., 2013). As the diving waves were the main driver of the Q-FWI process, we applied an inner and an outer mute to the data to isolate the diving 
waves. The inversion started at $3.5 \mathrm{~Hz}$ and we pushed it to $12 \mathrm{~Hz}$.

Similar to the approach used by Malinowski et al. (2011) and Xiao et al. (2018), the velocity model was updated down to the penetration depth of diving waves (around 3 $\mathrm{km}$ ), while the $\mathrm{Q}$ model was updated down to shallower depth ranges (around $500 \mathrm{~m}$ ). For the deeper section of the $\mathrm{Q}$ model, we used the result of $\mathrm{Q}$-tomography. This strategy comes from the observations that the most complex absorption anomalies are in the shallower layers and that the cross-talk between the two parameters might increase with depth. Moreover, the $Q$ model built by $Q$ tomography is more reliable in the deeper section, due to the limited diving-wave penetration.

\section{Results}

The field data are from a 3D seismic acquisition in the Foz do Amazonas basin, offshore Brazil. The seismic acquisition layout consists of a variable-depth streamer with twelve 8-km-long cables, and a nominal streamer separation of $100 \mathrm{~m}$. In this area, the water bottom varies from 80 to 150 meters.

The stratigraphic sequence of this area is characterized by lacustrine and fluvial sediments of the Rift phase (Cassiporé formation), followed by shales and sandstones during the passive margin phase (Limoeiro formation), then covered by shelf carbonates with very high velocity (Amapá formation). The shallower sediments above Amapá formation are characterized by shales and sandstones, cut by an intricate complex of paleo-channels as well as carbonate buildup features. These shallow carbonates most probably act as traps for the gas chimneys observed in the data, which are related to strong gas mobilization from the Amapá and Limoeiro formations.

Figure 1 shows the shallow portion (up to $500 \mathrm{~m}$ depth) of Q-PSDM sections migrated with the velocity and $Q$ inverted by Q-tomography (Figure $1 \mathrm{a}$ ) and by joint Q-FWI (Figure 1d). The velocity and 1/Q models overlaid on the Q-PSDM stacks are displayed respectively in Figures $1 \mathrm{~b}$ and $1 \mathrm{c}$ for the Q-tomography result, and in Figures $1 \mathrm{e}$ and If for the Q-FWI result. The same results for a crosssection in the same area are displayed in Figure 2.

For both velocity and $Q$, the tomography update of the shallow layer (Figures 1a-c and 2a-c) was challenging due to the limited illumination provided by reflection data. Despite only few traces being available for the residual move-out picking and the FPS estimation, the tomographic inversion was able to identify the presence of shallow anomalies characterized by low velocities and low $Q$ values, but with low resolution. There are still areas where the amplitude is under-compensated, since the shallow anomaly has a very complex geology which is challenging for the tomography to handle in the very shallow-water areas. We can observe that the joint Q-FWI update (Figures 1d-f and 2d-f) can better capture the geometry of the gas anomaly, while the lateral and vertical extension of the anomaly has good correlation with the seismic image, for both the velocity and $Q$ models. The imaging uplift brought by the $Q$ model and the velocity can be observed in Figures 1d and 2d: both the amplitude and the lateral continuity of the reflectors below the anomaly have been recovered.

Figure 3 shows the imaging improvements in the deeper section, with the events between the anomaly and the Amapá formation becoming sharper and having a better amplitude balance. Moreover, the reflectors inside the Amapá formation are better focused down to $3 \mathrm{~km}$ depth. Figures $3 \mathrm{a}$ and $3 \mathrm{~b}$ show the Q-PSDM migrated cross sections using the velocity and $Q$ inverted by $Q$ tomography. Figures $3 c$ and $3 d$ show the Q-PSDM migrated cross sections using the velocity and $Q$ inverted by joint $\mathrm{Q}-\mathrm{FWI}$. Although we limited the $\mathrm{Q}$ update only to the shallower portion of the model, still we can notice the benefit of the joint Q-FWI over the whole section. From the shallow to the deep layers, the images with Q-FWI models are able to capture and correct the absorption caused by the anomaly. Moreover, the events across and beneath the anomaly are better focused, with higher resolution and improved structural continuity.

In Figure 4, we show a depth slice at $720 \mathrm{~m}$. The image obtained with the Q-tomography model (Figure 4a) is still blurred in the absorption anomaly location (within the yellow oval). However, the result of Q-FWI (Figure 4b) shows an enhancement in the same location, where some events are now more continuous. The uplift brought by QFWI can also be evaluated by observing the better continuity of the events using a coherence attribute. In particular, we use the edge-stack attribute (Dorn \& Dominguez, 2017) which measures the coherency of the events for a given seismic volume. We can note that the edge-stack attribute with QFWI-based image has better coherency (Figure 4d) than that with Q-tomography (Figure 4c).

\section{Discussions}

Nowadays it is common to include FWI in the model building workflow for improving the resolution of the velocity model within the penetration depth of diving waves. In the results of the previous section, we show how the inclusion of $\mathrm{Q}$ in the $\mathrm{FWI}$ and the joint inversion of velocity and $Q$ models over a large frequency bandwidth can improve the imaging. Figure 5 shows a zoomed-in part (down to $1 \mathrm{~km}$ ) of the section displayed in Figure 3c. Figures $5 b$ and $5 c$ show a depth slice at $170 \mathrm{~m}$ of the velocity model inverted by the joint Q-FWI, with and without seismic stack overlay, respectively.

In the presence of absorptive bodies, it is important to invert for a spatially varying $Q$ model that better interprets the recorded data. In the frequency range used for FWI, the phase dispersion is the most prominent effect induced by $Q$ (Cheng et al., 2015). If an incorrect $Q$ is used and is not updated by the inversion process, any phase shift between modeled and observed data can only be 

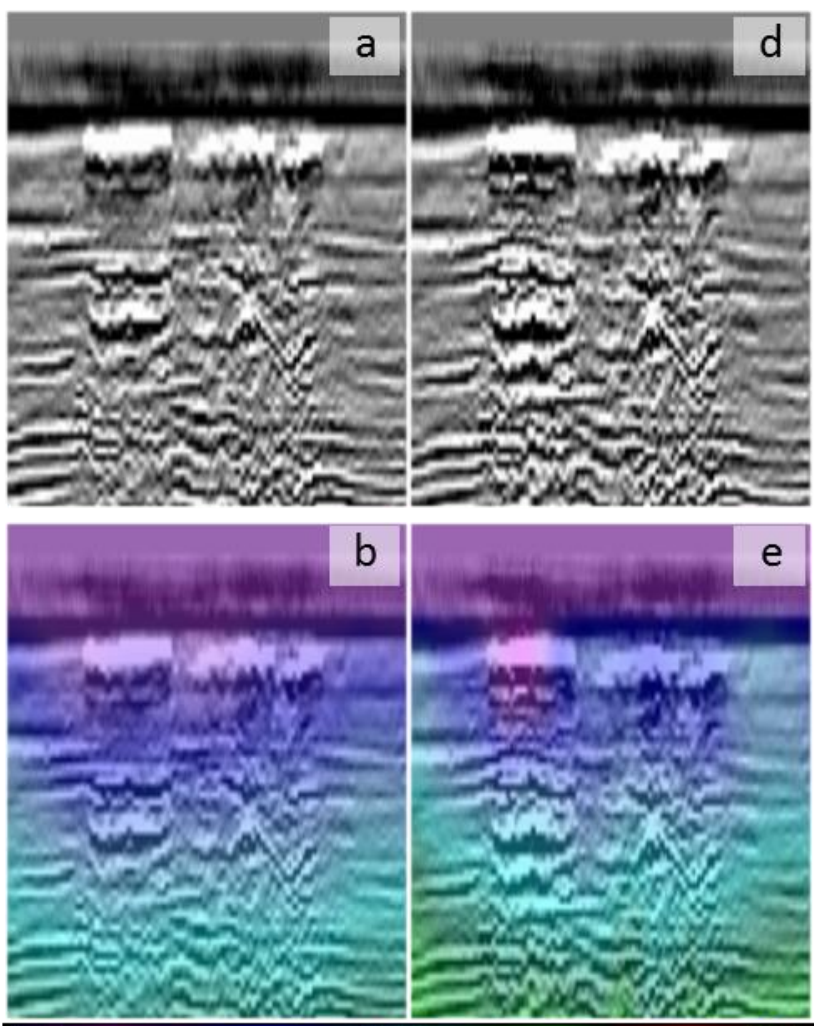

1.4

$\mathrm{km} / \mathrm{s}$
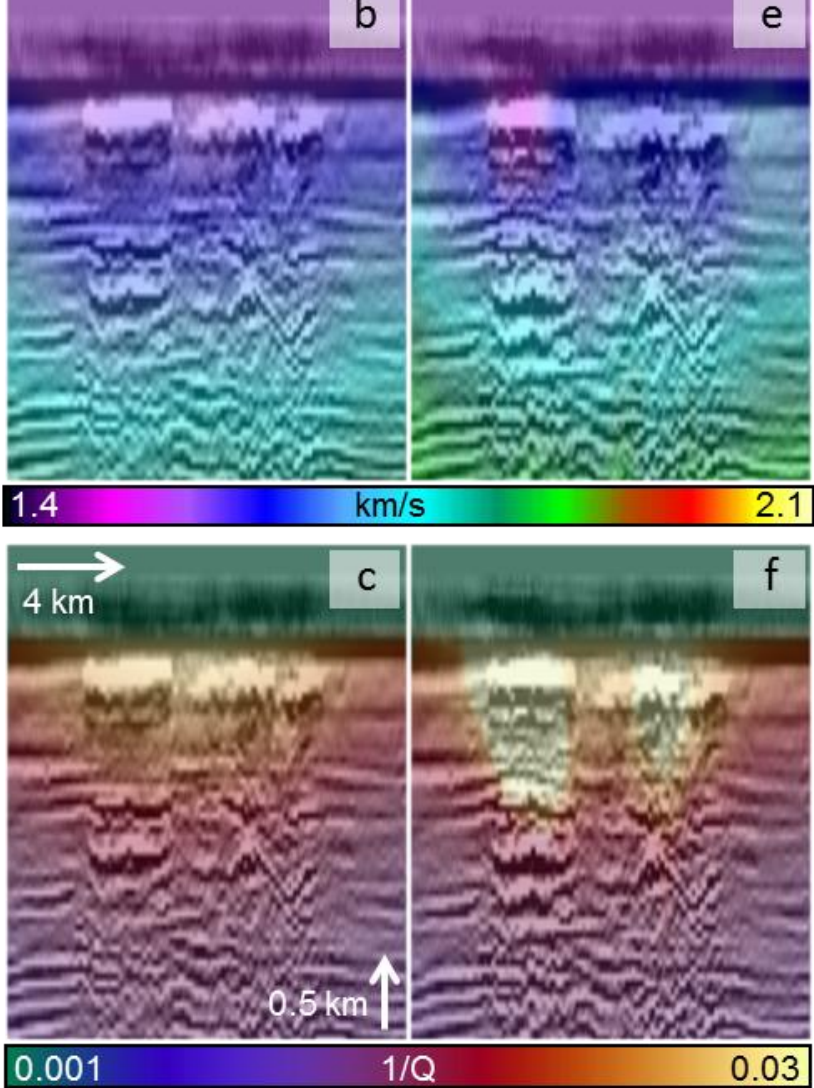

Figure 1 - Q-PSDM stack section down to $500 \mathrm{~m}$ depth migrated with (a) $Q$ and velocity model inverted with $Q$ tomography, and (d) $Q$ and velocity model inverted with joint Q-FWI. (b,c) Velocity and $1 / Q$ models overlaid seismic image inverted with $Q$-tomography. (e,f) Velocity and $1 / Q$ models overlaid seismic image inverted with Q-FWI.

compensated by a velocity update, thus possibly yielding an incorrect velocity update. The use of Q-FWI helps to mitigate the risk of compensating the absorption effect by an erroneous velocity update. Therefore, to avoid the cross-talk between these two parameters, it is important to correctly account for $Q$ in presence of strong absorption anomalies. In Figure $5 d$, we show the same section of Figure $5 \mathrm{a}$ migrated with the velocity model inverted by conventional FWI, without including Q. We can observe some undulations in the structures and artificial pull-ups
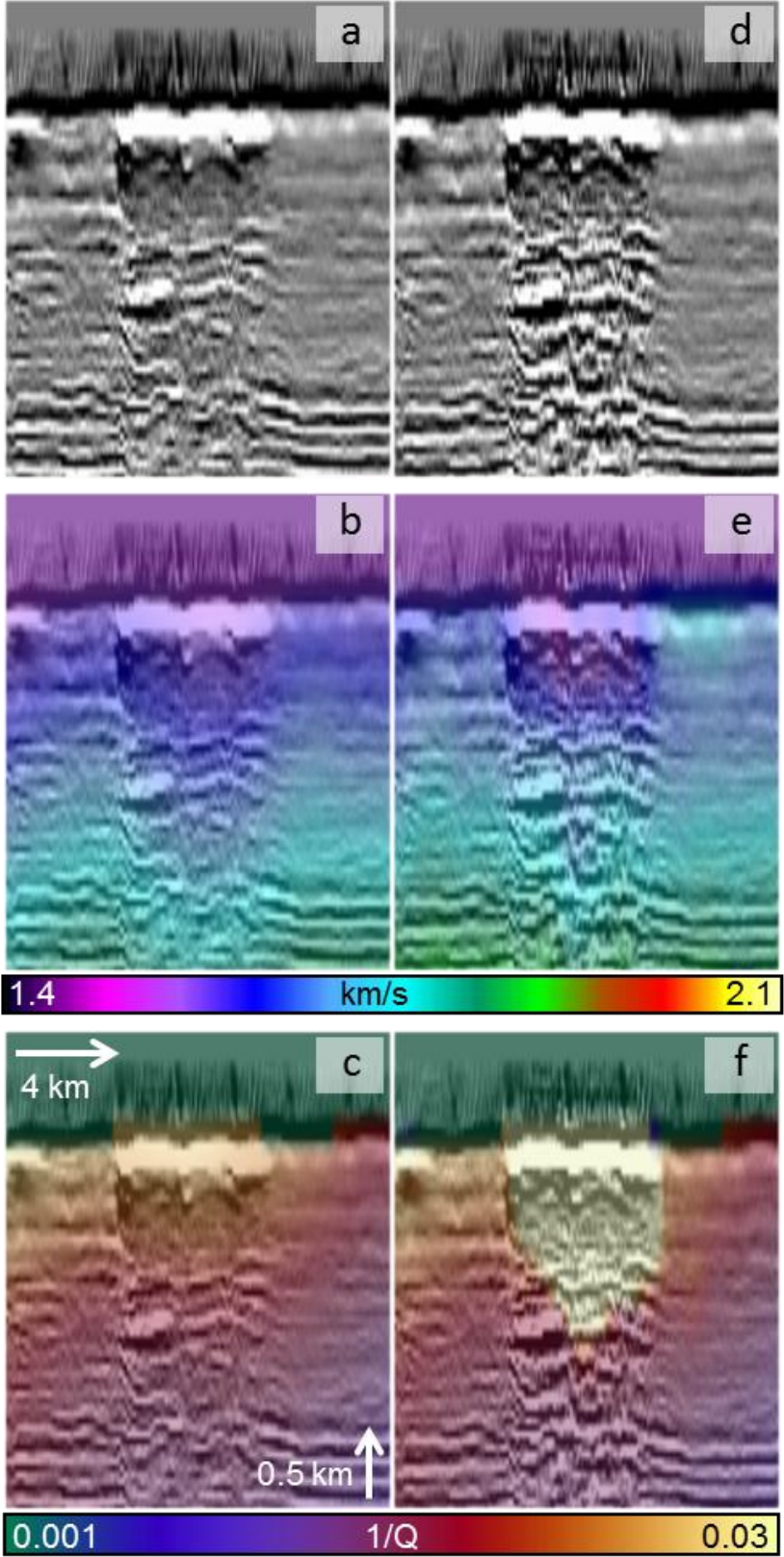

Figure 2 - Q-PSDM stack cross section down to $500 \mathrm{~m}$ depth migrated with (a) $Q$ and velocity model inverted with $Q$-tomography, and (d) $Q$ and velocity model inverted with joint Q-FWI. $(b, c)$ Velocity and $1 / Q$ models overlaid seismic image inverted with Q-tomography. (e,f) Velocity and 1/Q models overlaid seismic image inverted with Q-FWI.

and pull-downs that do not look geological. The same undulations are visible in the velocity models (Figures $5 \mathrm{e}$ and 5f). 

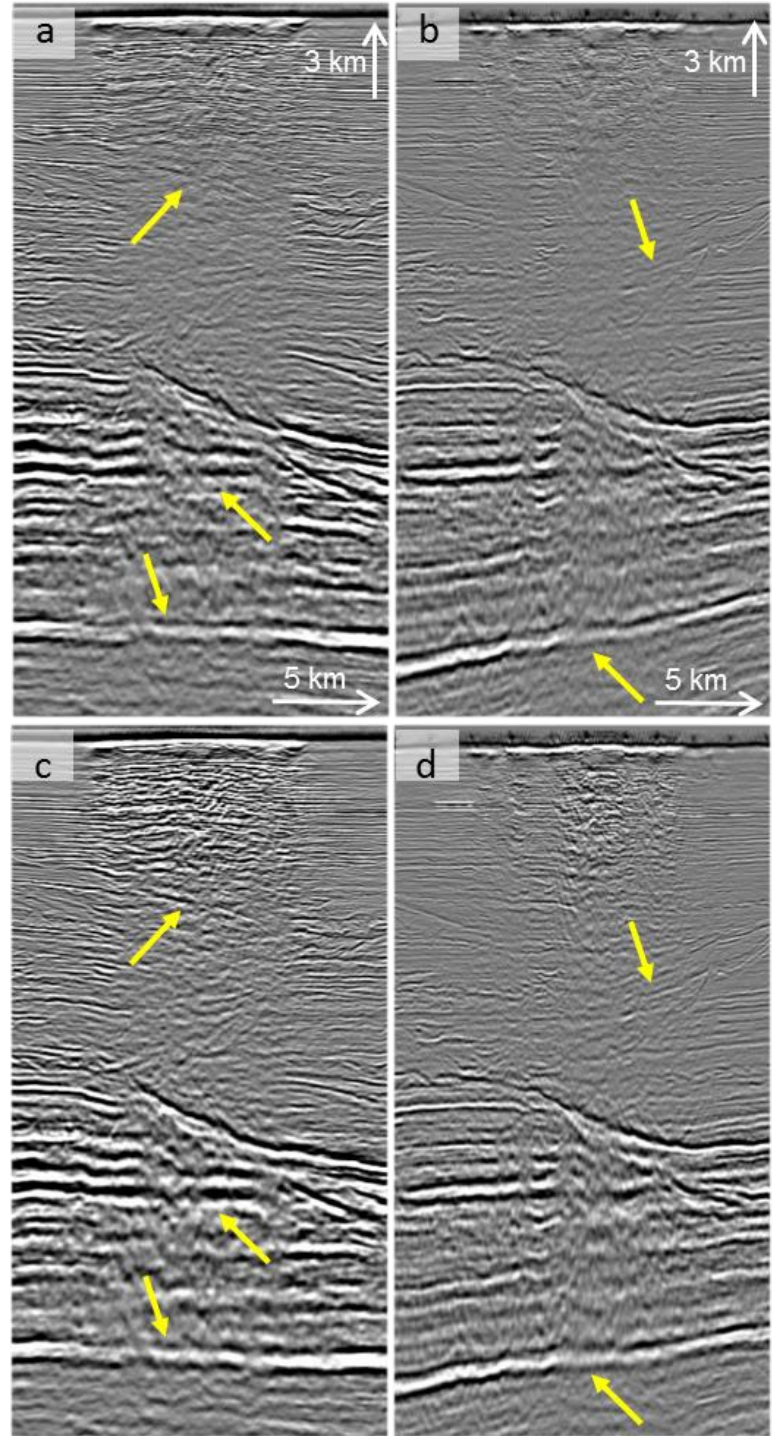

Figure 3 - Q-PSDM stack cross sections down to $3 \mathrm{~km}$ depth migrated with $(a, b) Q$ and velocity model inverted with $Q$-tomography, and $(c, d) Q$ and velocity model inverted with joint $Q-F W I$. The yellow arrows indicate improvements of the $Q-F W I$ results compared to the $Q$ tomography.

\section{Conclusions}

The shallow acquisition environment of this dataset affected the Q-tomography results due to the limited illumination provided by reflection data. The low-resolution tomographic $Q$ was not enough to solve the complex geometry of the absorptive anomalies. Instead, the divingwave-driven $\mathrm{Q}-\mathrm{FWI}$ was able to provide high-resolution models for both velocity and $\mathrm{Q}$. Despite the challenge of jointly inverting for these two parameters using FWI, we show that the use of Q-FWI over a relatively large range of frequencies yields an uplift in the Q-PSDM images.
We also discussed that it is important to jointly invert for velocity and $Q$ to avoid overfitting of one parameter into the other. More advanced imaging methods such as Q-RTM and least-squares Q-migration could be considered to further exploit the benefits of the joint $\mathrm{Q}-\mathrm{FWI}$ inverted models.

\section{Acknowledgments}

The authors would like to thank CGG for the permission to publish this work and CGG Multi-Client \& New Ventures for the Foz do Amazonas data set.

\section{References}

CHENG, X., K. JIAO, D. SUN, AND D. VIGH, A new approach of visco-acoustic waveform inversion in time domain: 85th Conference \& Exhibition, SEG, Expanded Abstract, 1183-1187, 2015.

DORN, G. A. AND J. P. DOMINGUEZ, Post-stack attribute-based fracture characterization: A case study from the Niobrara shale: First Break, 35, 85-89, 2017.

GAMAR, F., P. GUILLAUME, A. PICA, G. PIGNOT, P. POGGI, A. HENRY-BAUDOT, A. PRESCOTT, A. GACHA, D. CAROTTI, AND V. PRIEUX, Automatic gas pockets detection by high-resolution volumetric Q-tomography using accurate frequency peak estimation: 77th Conference and Exhibition, EAGE, Extended Abstracts, Th-N107-10, 2015.

GUILLAUME, P., S. HOLLINGWORTH, X. ZHANG, A. PRESCOTT, R. JUPP, G. LAMBARÉ, AND O. PAPE, Multi-layer tomography and its application for improved depth imaging: 82nd Conference \& Exhibition, SEG, Expanded Abstracts, 1-5, 2012.

HUNG, B., K. F. XIN, S. BIRDUS, AND J. SUN, 3-D tomographic amplitude inversion for compensating transmission losses in the overburden: 70th Conference and Exhibition, EAGE, Extended Abstracts, H004, 2008.

MALINOWSKI, M., S. OPERTO, AND A. RIBODETTI, High-resolution seismic attenuation imaging from wideaperture onshore data by visco-acoustic frequency-domain full-waveform inversion: Geophysical Journal International, 186(3), 1179 - 1204, 2011.

POOLE, G., C. DAVISON, J. DEEDS, K. DAVIS, AND G. HAMPSON, Shot-to-shot directional designature using near field hydrophone data: 83rd Annual International Meeting, SEG, Expanded Abstracts, 4236-4240, 2013.

PLESSIX, R. E., A. STOPIN, H. KUEHL, V. GOH, AND K. OVERGAAG, Visco-acoustic full waveform inversion: 78th Conference and Exhibition, EAGE, Extended Abstracts, Tu-SRS2-04, 2016. 
STOPIN, A., R. E. PLESSIX, H. KUEHL, V. GOH, AND K. OVERGAAG, Application of visco-acoustic full waveform inversion for gas cloud imaging and velocity model building: 78th Conference and Exhibition, EAGE, Extended Abstracts, Tu-STZ0-12, 2016.

WANG, M., Y. XIE, B. XIAO, A. RATCLIFFE, AND T. LATTER, Visco-acoustic full-waveform inversion in the presence of complex gas clouds: 88th Conference \& Exhibition, SEG, Expanded Abstracts, 5516-5520, 2018.

XIAO, B., A. RATCLIFFE, T. LATTER, Y. XIE, AND M. WANG, Inverting near-surface absorption bodies with fullwaveform inversion: a case study from the North Viking Graben in the Norwegian North Sea: 80th Conference and Exhibition, EAGE, Extended Abstracts, A-12-03, 2018.

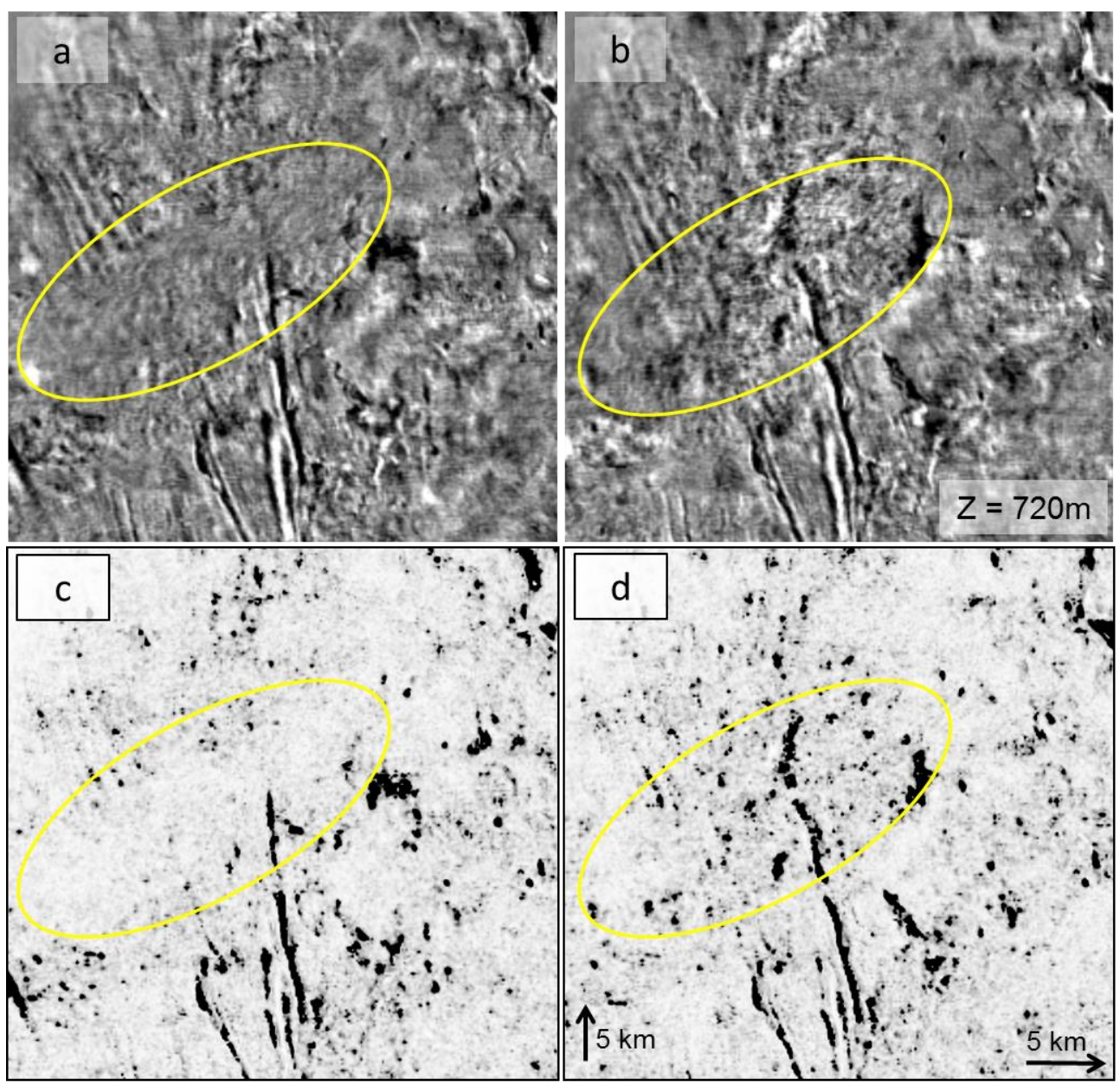

Figure 4-Q-PSDM depth slice at $720 \mathrm{~m}$ depth migrated with (a) $Q$ and velocity model inverted with $Q$-tomography, and (b) $Q$ and velocity model inverted with joint $Q$-FWI. Stack edge attribute extracted at $720 \mathrm{~m}$ depth using (c) the $Q$ tomography migrated image and (d) the Q-FWI migrated image. The yellow oval indicates the location of the absorption anomaly. 

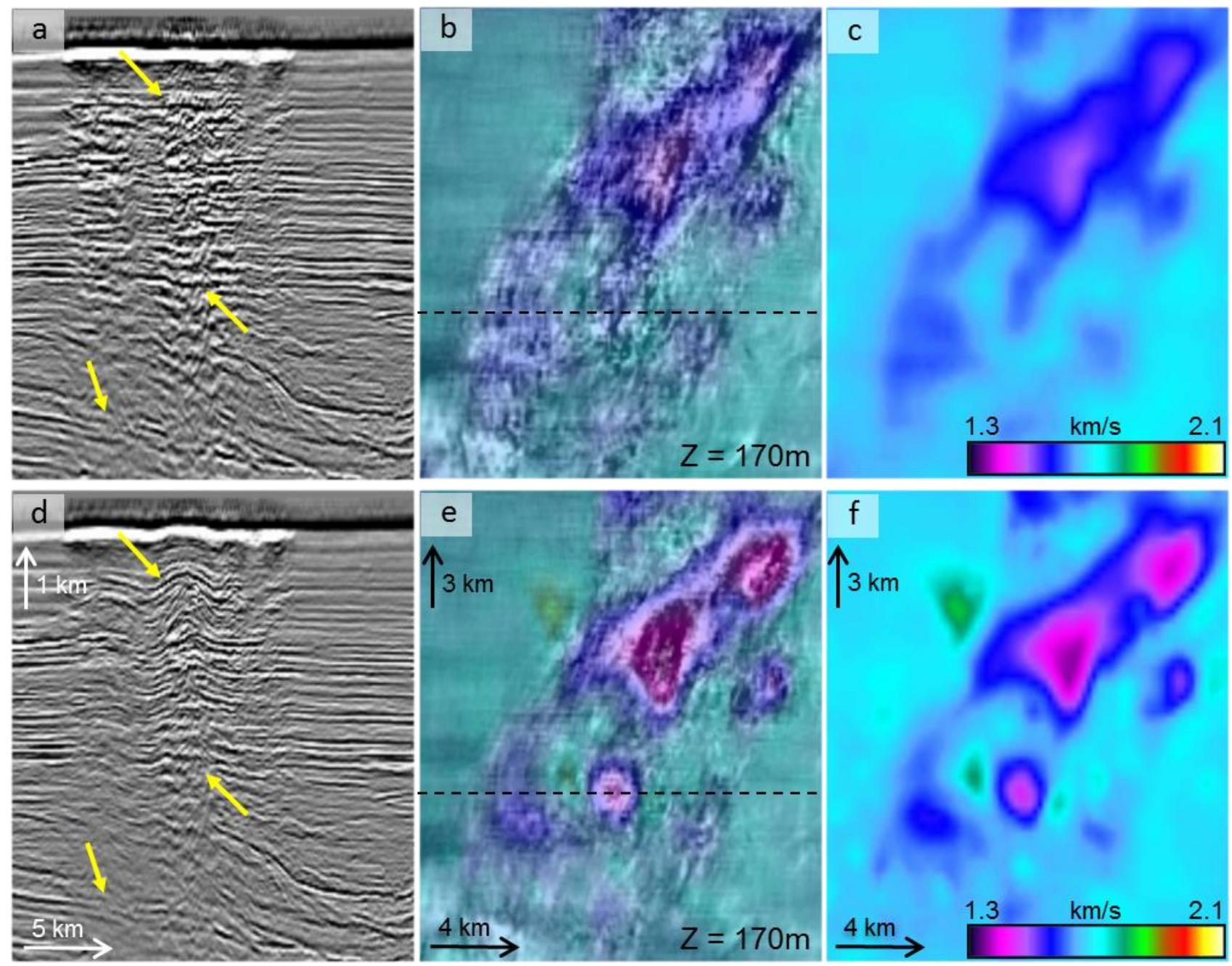

Figure 5-Q-PSDM stack section (same as shown in Figure 3) down to $1 \mathrm{~km}$ depth using (a) the velocity model inverted by $Q-F W I$ and (d) the velocity model inverted by conventional FWI. For display purposes, the same $Q$ amplitude compensation is applied to both images (a) and (d). Yellow arrows indicate location of improvements when using the joint Q-FWI, compared with the conventional FWI. Depth slice of the velocity model inverted by Q-FWI with (b) and without (c) seismic overlay. Depth slice of the velocity model inverted by conventional FWI with (e) and without (f) seismic overlay. The dashed black line indicates the position of the section. 